\title{
How Equilibrium Prices Reveal Information in Time Series Models with Disparately Informed, Competitive Traders
}

\author{
Todd B. Walker \\ Indiana University Bloomington
}

September 22, 2006

This paper can be downloaded without charge from the Social Science Research Network electronic library at: http://ssrn.com/abstract=932695.

The Center for Applied Economics and Policy Research resides in the Department of Economics at Indiana University Bloomington. CAEPR can be found on the Internet at:

http://www.indiana.edu/-caepr. CAEPR can be reached via email at caepr@indiana.edu or via phone at 8D-855-4050.

(02006 by Todd B. Walker. All rights reserved. Short sections of text, not to exceed two paragraphs, may be quoted without explicit permission provided that full credit, including $\odot$ notice, is given to the source. 


\title{
How Equilibrium Prices Reveal Information in Time Series Models With Disparately Informed, Competitive Traders*
}

\author{
Todd B. Walker ${ }^{\dagger}$ \\ Indiana University
}

February 2006

\begin{abstract}
Accommodating asymmetric information in a dynamic asset pricing model is technically challenging due to the problems associated with higher-order expectations. That is, rational investors are forced into a situation where they must forecast the forecasts of other agents. In a dynamic setting, this problem telescopes into the infinite future and the dimension of the relevant state space approaches infinity. By using the frequency domain approach of Whiteman (1983) and Kasa (2000), this paper demonstrates how information structures previously believed to preserve asymmetric information in equilibrium, converge to a symmetric information, rational expectations equilibrium. The revealing aspect of the price process lies in the invertibility of the observed state space, which makes it possible for agents to infer the economically fundamental shocks and thus eliminating the need to forecast the forecasts of others.
\end{abstract}

Keywords: Asset Pricing, Asymmetric Information

JEL Classification Numbers: G12, D82

\footnotetext{
*I would like to thank Matthew Billett, John Geweke, M. Beth Ingram, Ken Kasa, Narayana Kocherlakota, Matthew Pritsker, B. Ravikumar, Gene Savin, Gábor Virág, and participants at New York University, the Federal Reserve Board of Governors, Indiana University, Miami University, the University of Iowa Alumni Conference, the Midwest Macroeconomics Conference, and the Midwest Economic Theory Group for their helpful comments and suggestions. I would especially like to thank Chuck Whiteman for many insightful discussions. All remaining errors are my own.

${ }^{\dagger}$ First version: January 2005. Correspondence to: walkertb@indiana.edu
} 


\section{Introduction}

Keynes (1936) likened investment decisions to beauty contests in which competitors had to select the "prettiest faces" from a hundred photographs. Keynes argued that competitors will not pick the faces they find most attractive but will be forced into a situation where they must guess what average opinion expects the average opinion to be; that is, they must form higher-order expectations. This scenario accurately describes the behavior of differentially informed investors in a simple asset pricing model. Traders recognize that information disseminated through current and past prices reflects not only the information endowment of other investors, but also their attempts to forecast the forecasts of other traders. The goal of this paper is to explicitly model how information is disseminated through prices, and to show when this dissemination becomes too widespread to maintain asymmetric information in equilibrium. Models previously believed to impose asymmetric information in equilibrium (e.g., Singleton (1987)) are shown to converge to symmetric information, representative agent models.

The empirical failures of representative agent models have inspired a vast literature that introduces information heterogeneity and higher-order expectations in hopes of reconciliating theory with data. ${ }^{1}$ However, the computational problems associated with heterogeneous information have long proven to be a significant impediment to solving dynamic, intertemporal trading models with differentially informed investors. The crux of the problem is how to model higher-order expectations. Investors who anticipate what average opinion expects the average opinion to be form, what Keynes called, third-degree expectations. Due to the complexities of the fourth degree, Phelps (1983) quipped "one gets a vertiginous feeling, the eyes dull, and the face goes slack." Yet in a dynamic model, rational investors will fall into the trap of infinite regress where they must forecast what the average opinion expects the average opinion expects the average opinion..., ad infinitum. Agents behaving optimally must incorporate these infinite-order expectations into their beliefs, thus making the state space infinite dimensional.

To circumvent the problem of infinite regress, existing models typically make simplifying assumptions or cleverly construct the model to bypass infinite regress. ${ }^{2}$ Lucas (1975) was the first to capture the technical formalities of infinite regress in expectations, yet neatly sidestepped the problem by assuming traders got together each period and pooled their forecasts. Townsend (1983) did not make this simplifying assumption and resorted to a truncation strategy, where the state of the economy is revealed to all agents with a two-period lag. Singleton (1987) was the first to study a dynamic asset pricing model with higher-order belief dynamics and also employed the truncation

\footnotetext{
${ }^{1}$ Recent contributions include Angeletos and Pavan (2005), Allen, Morris, and Shin (2005), Bacchetta and van Wincoop (2004), Hellwig and Veldkamp (2005), Morris and Shin (2003), Nimark (2005), Ui (2003), and Woodford (2003).

${ }^{2}$ Approaches to elude the problem of infinite regress have included: constructing a hierarchical information structure with limited dispersion of information (e.g., Wang (1994), Wang (1993)); assuming a continuum of investors and invoking the law of large numbers to remove all aggregate uncertainty (e.g., He and Wang (1995)); assuming a finite or static environment in which the asset is liquidated at a certain date (e.g., Allen, Morris, and Shin (2005), Foster and Viswanathan (1996)).
} 
technique of Townsend to solve the model. ${ }^{3}$ In contrast, this paper does not assume away the infinite-regress problem and demonstrates how frequency domain techniques can be used to solve dynamic, asymmetric information models. Once an analytical solution to the model is derived, the size of the approximation error associated with the popular truncation technique can be assessed. One of the main results of this paper is to demonstrate how the Townsend-Singleton truncation technique can lead to spurious results.

While couched within the context of an asset pricing model, the techniques introduced here contribute more broadly to the literature on solving dynamic models with asymmetric information. This paper builds on the work of Sargent (1991), Kasa (2000), and Pearlman and Sargent (2005). Sargent (1991) describes a method for computing equilibrium (vis-á-vis Townsend's partial revelation approach) in which the state of the economy is never revealed to the agents. Kasa (2000) demonstrates how the frequency domain significantly decreases the computational cost of Sargent's solution method. More recently, Pearlman and Sargent (2005) show that the signal extraction problem of Townsend (1983) is not enough to preserve divergent beliefs when agents act rationally. By defining a new state variable (the agent's forecast error) and applying the recursive methods of Pearlman, Currie, and Levine (1986), they show that every agent will make the same forecast in equilibrium, thus eliminating the need to forecast the forecasts of others. However, these advancements have all been made in the context of Townsend (1983), which is a special case due to the unique structure of the model. ${ }^{4}$ But it has long been suspected that this conclusion applies more generally to a broader class of models. Consider the concluding remarks of Singleton (1987),

"Another interesting finding is that the equilibrium prices for the models with disparate information and partial, homogeneous information follow very similar time series properties. It remains to be seen whether this carries over to alternative parameterizations and information structures. Based on the findings to date, however, it appears that disparate information per se in a competitive market does not significantly effect the equilibrium price process."

The contribution of this paper is to show how a certain class of signal extraction problems does not generate and preserve divergent beliefs in a dynamic equilibrium when agents can learn from past forecast errors. While this has long been believed to be true, no formal analysis to date has proven this claim. Working within the framework of Singleton's bond market model, I use frequency domain techniques to show how endogenous variables reveal all privately held information. This, in turn, eliminates the need to forecast the forecasts of others and the asymmetric information "equilibrium" will be shown to converge to a symmetric information equilibrium. The proof of the convergence result assumes that agents are equipped with basic econometric knowledge. The results

\footnotetext{
${ }^{3}$ More recently, Bacchetta and van Wincoop (2004) use Townsend's truncation technique to analyze exchange rate dynamics.

${ }^{4}$ The model of Townsend induces informational asymmetries by assuming that prices do not simultaneously clear markets due to production lags. Thus the resulting signal extraction problem is endemic to the economy (see, Kasa (2000)) and is unlike the "typical" signal extraction problem (i.e., where there are more noises than signals).
} 
derived here have implications for static models of asymmetric information, models that assume agents have limited information capacity, and models that use the Townsend-Singleton truncation technique.

The next section introduces the bond market model of Singleton (1987). Section 3 provides a solution method for dynamic models of asymmetric information, solves the model in a symmetric information setting and in an asymmetric information environment, and proves that these two information structures yield the same equilibrium.

\section{The Model}

In this section, I briefly outline the model of Singleton, which was motivated by the market microstructure of the U.S. bond market. ${ }^{5}$ The basic features of the model include a competitive, Walrasian market structure with a single asset that is traded among speculative and nonspeculative or liquidity traders.

Suppose there is a continuum of investors indexed by $i \in[0,1]$, and each trader invests in a single risky security with price $p_{t}$ and stochastic coupon payment $c_{t}$ at date $t$. The coupon stream $\left\{c_{t}\right\}$ is assumed to be normally distributed and to follow a first-order autoregressive process

$$
c_{t}=\bar{c}+\psi c_{t-1}+u_{t}, \quad \mathbb{E}\left(u_{t}\right)=0, \operatorname{var}\left(u_{t}\right)=\sigma_{u}^{2}, \quad|\psi|<1
$$

Purchases of the security are financed by borrowing at the constant rate $r$. Therefore, the wealth of trader $i$ evolves according to

$$
w_{i, t+1}=z_{i t}\left(p_{t+1}+c_{t+1}\right)-(1+r)\left(z_{i t} p_{t}-w_{i t}\right)
$$

where $z_{i t}$ denotes the holdings of the risky asset at date $t$. The $i$ th investor is assumed to have a one-period investment horizon and to rank alternative investment strategies according to the negative-exponential utility function

$$
\mathbb{E}_{t}^{i}-\exp \left(-\gamma w_{i, t+1}\right)
$$

where $\mathbb{E}_{t}^{i}$ denotes the expectation of investor $i$ conditioned on his information set $\Omega_{t}^{i}$ at date $t$, and $\gamma$ is the constant coefficient of absolute risk aversion that is common to all traders. Given the assumption that underlying sources of uncertainty are normally distributed, it is well known that wealth maximization implies the following demand schedule for the risky asset

$$
z_{i t}=\frac{\mathbb{E}_{t}^{i}\left(p_{t+1}+c_{t+1}\right)-\alpha p_{t}}{\gamma \delta_{i}}
$$

\footnotetext{
${ }^{5}$ The literature has shown a renewed interest in Singleton (1987) due to the work of Morris and Shin (2003) and Allen, Morris, and Shin (2005). The Allen-Morris-Shin framework is a static, three-period asset pricing model. The techniques of Singleton (1987) can be used to extend the Allen-Morris-Shin setup to an infinite horizon economy.
} 
where $\delta_{i}=\operatorname{var}_{t}^{i}\left(p_{t+1}+c_{t+1}\right)$ and $\alpha=(1+r)>1$. It is the presence of higher-order moments $\delta_{i}$ that lead to multiple equilibria. Since the goal of this paper is to keep track of how information disseminates through asset prices, I will normalize the coefficient of risk aversion and the variance parameter to unity. The resulting demand schedule

$$
z_{i t}=\mathbb{E}_{t}^{i}\left(p_{t+1}+c_{t+1}\right)-\alpha p_{t}
$$

not only offers a unique equilibrium, but also has a broad appeal in that there are many economic models that have equations analogous to that of (2.2). For example, with $c_{t+1}$ defined as dividends, we have a present value model for stock prices; with $c_{t+1}$ defined as the difference between national money supplies and income levels, it becomes the monetary model of exchange rates; with $c_{t+1}$ defined as a short-term interest rate, it becomes the expectations hypothesis of the term structure; and so on. Appendix A solves the model without this simplifying assumption.

Singleton assumed supply of the asset was stochastic and determined by nonspeculative traders, which serves to break the no-trade theorem. That is, the net supply of the asset $s_{t}$ (total supply less nonspeculative demand at time $t$, less the mean difference) is the sum of two stochastic components and price

$$
s_{t}=A(L) \varepsilon_{1 t}+B(L) \varepsilon_{2 t}+\xi p_{t}
$$

where $A(L)$ and $B(L)$ are (possibly infinite-order) polynomials in nonnegative powers of the lag operator $L$ with square-summable coefficients (i.e., $\left.\sum_{j=0}^{\infty} A_{j}^{2}<\infty\right)$, and $\left\{\varepsilon_{1 t}\right\}$ and $\left\{\varepsilon_{2 t}\right\}$ are mutually and serially uncorrelated, normally distributed random variables with zero mean and variance components $\sigma_{\varepsilon_{1}}^{2}, \sigma_{\varepsilon_{2}}^{2}{ }^{6}{ }^{6}$ The net supply is interpreted as arising from non-speculative traders in the U.S. bond market (e.g., the U.S. Treasury, the Federal Reserve, financial intermediaries). The shocks to net supply could thus arise from non-speculative traders attempting to satisfy "macroeconomic" objectives and for technical reasons related to the financial intermediation process. Non-speculative traders are assumed to respond positively to an increase in price; thus, following Singleton, I will assume $\xi>1$.

The market clearing condition equates equations (2.2) and (2.3), and gives the equilibrium price to be

$$
\varphi p_{t}=\int_{0}^{1} \mathbb{E}_{t}^{i} p_{t+1} d i+\bar{c}+\psi c_{t}-s_{t}
$$

where $\varphi=(\xi+\alpha)>1$. Thus, the equilibrium price of the asset at time $t$ depends upon the market-wide average expectation of the asset at $t+1 .^{7}$ However, each trader's forecast of $p_{t+1}$ will depend upon the market-wide forecast of $p_{t+2}$, and so on, ad infinitum. If traders' information sets

\footnotetext{
${ }^{6}$ Equation (2.3) places no restrictions on the serial correlation properties of $\left\{s_{t}\right\}$. The Wold Decomposition Theorem allows for such a general structure (see, Sargent (1987)).

${ }^{7}$ Notice that equation (2.4) does not imply asymmetric information with respect to the coupon stream. The argument is that asymmetric information in the U.S. bond market would arise with respect to the supply process.
} 
generate disparate expectations, the problem of infinite regress arises. When forecasting $p_{t+1}$, each trader must take into account every other traders' forecast of $p_{t+1}, p_{t+2}, \cdots$. If the equilibrium price does not reveal all privately held information, then the usual method of solving for the rational expectations equilibrium (e.g., Blanchard and Kahn (1980)) breaks down. ${ }^{8}$ The principal technical difficulty is that agents are extracting signals from endogenous variables. When endogenous variables convey information, it becomes difficult to identify a tractable set of state variables because an agent's notion of the state of the economy would include other agents' forecasts of the asset price at indefinitely many future dates, thereby making the dimension of the state indefinitely large. In contrast, for symmetric information, representative agent models involving signal extraction from endogenous variables, the true state vector is latent from the optimizer. Beliefs then serve as the defacto state variable (e.g., Muth (1960)), and the Kalman filter can be employed to estimate the hidden state. But when the state becomes infinite dimensional, as it does here, this method cannot be applied.

The next section describes two methods for handling the infinite-dimensional state space. One solution method is the Townsend-Singleton truncation technique, which assumes that asymmetric information only persists for a finite number of periods. The second solution technique uses frequency domain methods to solve for equilibrium without relying on truncating the state space. Following this solution method, one can then give conditions under which the equilibrium price process contains too much information to preserve asymmetric information in equilibrium.

\section{Information and Solution Techniques}

In this section, I first describe the basic solution technique used to solve the model and do so under the assumption of a homogenous information structure. I then give conditions under which the asymmetric informational setup of Singleton converges to this homogenous, representative agent economy. Finally, I demonstrate how the Townsend-Singleton truncation technique can lead to substantial approximation error.

\subsection{Solution Method}

It is important to be meticulous about the conditioning information sets of agents in models of asymmetric information, especially when information can be extracted from endogenous variables. To place structure on the problem, solutions to the model will be sought in the space spanned by square-summable linear combinations of the fundamental driving processes $\left\{u_{t}, \varepsilon_{1 t}, \varepsilon_{2 t}\right\}$. This assumption rules out sunspot equilibria and implies that the solution will lie in a well-known Hilbert space (i.e., the space of square-summable sequences, denoted $\ell_{2}(-\infty, \infty)$ ). The information set of agent $i$ at time $t$, denoted by $\Omega_{t}^{i}$, represents the current and past values of the variables observed by

\footnotetext{
${ }^{8}$ Moreover, as recently emphasized by Allen, Morris, and Shin (2005), average expectation operators usually fail to satisfy the law of iterated expectations. Therefore, in an economy with asymmetric information, the price of an asset today will not equal the representative agent's discounted expected value of the asset's payoff stream conditional on information available today, but the price will also encompass investors' higher-order beliefs.
} 
trader $i$. There will be two types of information available to each agent - exogenous and endogenous. Exogenous information is by definition not affected by market forces and will be denoted by $U_{t}^{i}$. Endogenous information may be generated through market interactions of differentially informed agents.

Remark 1. Assuming all agents behave rationally, the Projection Theorem (see Brockwell and Davis (1991)) implies that agent $i$ 's conditional expectation of $p_{t+1}$ is the orthogonal projection of $p_{t+1}$ on the smallest closed subspace which contains $\Omega_{t}^{i}$. This subspace will include both exogenous and endogenous information, and since the collection of variables is jointly normal, conditional expectations will reduce to linear least-squares projections. Let $H_{x}(t)$ denote the space spanned by square-summable linear combinations of current and past values of $x$. Then trader $i$ 's expectation of $p_{t+1}$ is given by

$$
\mathbb{E}_{t}^{i}\left(p_{t+1}\right)=\Pi\left[p_{t+1} \mid \Omega_{t}^{i}\right]=\Pi\left[p_{t+1} \mid U_{t}^{i} \bigvee H_{p}(t)\right]
$$

where $\Pi$ denotes linear least-squares projection and $U_{t}^{i} \bigvee H_{p}(t)$ is standard notation for the "linear space spanned by $U_{t}^{i}$ and $H_{p}(t) . "$

Agents use all exogenous information and information generated by current and past values of the equilibrium price in evaluating the expectation of tomorrow's price. The expectation of investor $i$ is then simply the orthogonal projection of $p_{t+1}$ onto the subspace generated by $U_{t}^{i}$ and $H_{p}(t)$. Let $\Xi_{t}$ denote the set of information known by all traders at date $t$ (i.e., $\Xi_{t} \equiv \bigcup_{i} \Omega_{t}^{i}$ ). We are now ready to define a rational expectations equilibrium (REE).

Definition 1. A rational expectations equilibrium is a stochastic process for $\left\{p_{t}\right\}, p_{t} \in \Xi_{t}$, that satisfies market clearing (2.4), where expectations are formed according to (3.1).

The requirement, $p_{t} \in \Xi_{t}$, is what Futia (1981) referred to as the axiom of "no divine revelation." This condition prevents equilibrium prices at date $t$ from conveying any more information than that which could be in principle be available to traders at date $t$. In a symmetric equilibrium, agents' expectations coincide:

Definition 2. We say a REE is symmetric if after observing the history of equilibrium prices $\left\{p_{t-j}\right\}_{j=0}^{\infty}$ all traders have identical information and make the same forecasts. That is

$$
\mathbb{E}_{t}^{i}\left(p_{t+1}\right)=\Pi\left[p_{t+1} \mid U_{t}^{i} \bigvee H_{p}(t)\right]=\mathbb{E}_{t}^{j}\left(p_{t+1}\right)=\Pi\left[p_{t+1} \mid U_{t}^{j} \bigvee H_{p}(t)\right]
$$

for all $i$ and $j$.

The equilibrium of the model is computed as follows. First, each trader uses all available information at time $t\left(\Omega_{t}^{i}\right)$ to form beliefs about the current price process. Second, the conditional expectation of $p_{t+1}$ will be taken via Wiener-Kolmogorov optimal prediction formulas. Third, the appropriate form of equation (2.4) is then used to impose market clearing. In solving the subsequent 
fixed-point problem, I will appeal to the Riesz-Fischer Theorem and derive the solution in the frequency domain. ${ }^{9}$ The benefit of working in the frequency domain is computational convenience. Working in the time domain, Sargent (1991) shows how to convert the infinite dimensional state space associated with the forecasting the forecasts of others problem of Townsend (1983) into a finite dimensional system. Rather than guessing an infinite-order autoregressive representation for beliefs (the state variable), Sargent models agents as forecasting by fitting low-order autoregressive, moving-average (ARMA) representations. By introducing moving-average components in agents' perceptions, Sargent utilizes the ability of low-order ARMA representations to replicate the space of some infinite-order AR representations. The drawback of this approach is that not only does one have to solve a fixed point problem in the coefficients of the ARMA process, but the order of the ARMA process must be matched as well. This led to the insight of Kasa (2000), who shows how this two-step process can be condensed into a single step by working in the frequency domain. As opposed to guessing a functional form for beliefs, applying the Kalman filter, and then attempting to match coefficients, the frequency domain allows one to work with a functional fixed point problem. Therefore coefficients and order of the ARMA process are matched simultaneously by using the theory of the residue calculus. However, this process will only generate a candidate equilibrium price process. Traders will surely condition on past prices, therefore in order for the information structure to preserve disparate expectations, the equilibrium price process must not reveal other agents' privately held information. In a sense, we must have an "informational fixed point" in order for the price process to be sustainable in equilibrium. If the candidate equilibrium price does reveal information to agents that they did not have in forming expectations at step one, then the process just described will be repeated with the updated information sets of the traders. It is this final step of the solution method that is the main contribution of the paper.

\subsection{Homogenous Information}

As a baseline model, it is useful to first assume a symmetric information structure that avoids infinite regress. ${ }^{10}$ To this end, suppose that every investor observes past prices, the coupon stream, and net supplies. That is, the common information set of every trader $i$ is given by

$$
\Omega_{t}^{i}=\left\{p_{t-j}, c_{t-j}, s_{t-j}: j \geq 0\right\} \quad \forall i
$$

Given this informational assumption, an investor's belief about the average equilibrium price will be represented by a linear combination of current and past values of $\left\{p_{t}, c_{t}, s_{t}\right\}$. In the absence of sunspots, these stochastic processes will be driven by the underlying shocks $\left\{u_{t}, \varepsilon_{1 t}, \varepsilon_{2 t}\right\}$. For reasons outlined in Whiteman (1983), it is much simpler to calculate equilibrium prices and quanti-

\footnotetext{
${ }^{9}$ Recall the Riesz-Fischer Theorem states there is an equivalence (i.e., an isometric isomorphism) between the space of square-summable sequences denoted by $\ell_{2}(-\infty, \infty)$ and the space of square integrable functions, $L^{2}[\pi,-\pi]$; the former is referred to as the time domain and the latter the frequency domain.

${ }^{10}$ This case will not only serve as a benchmark but the next section shows how ex ante disparate information structures can degenerate to this homogeneous information case.
} 
ties if agents' expectations are computed under the assumption that they can see these underlying shocks. This "candidate" equilibrium will be realizable if and only if the space spanned by current and past values of the candidate processes $\left\{c_{t}, s_{t}, p_{t}\right\}$ is identical to that spanned by current and past values of the underlying shocks $\left\{u_{t}, \varepsilon_{1 t}, \varepsilon_{2 t}\right\}$. Therefore, trader $i$ 's expectation of $p_{t+1}$ is assumed to be given by

$$
\mathbb{E}_{t}^{i}\left(p_{t+1}\right)=\Pi\left[p_{t+1} \mid H_{s}(t) \bigvee H_{p}(t) \bigvee H_{c}(t)\right]=\Pi\left[p_{t+1} \mid H_{u}(t) \bigvee H_{\varepsilon_{1}}(t) \bigvee H_{\varepsilon_{2}}(t)\right]
$$

Thus we proceed by assuming agents see the economic shocks, compute a candidate equilibrium under that assumption, and then check to verify that the candidate equilibrium process $\left\{c_{t}, s_{t}, p_{t}\right\}$ would enable agents to uncover $\left\{u_{t}, \varepsilon_{1 t}, \varepsilon_{2 t}\right\}$. If this recovery is possible, then the corresponding equilibrium is a rational expectations equilibrium (REE).

Thus, step one of the solution process suggests we begin with the tentative assumption that every trader believes the average equilibrium price to be given by

$$
p_{t}=\sum_{j=0}^{\infty} G_{j} u_{t-j}+\sum_{j=0}^{\infty} D_{j} \varepsilon_{1, t-j}+\sum_{j=0}^{\infty} F_{j} \varepsilon_{2, t-j} \equiv G(L) u_{t}+D(L) \varepsilon_{1 t}+F(L) \varepsilon_{2 t}
$$

where $G(L), D(L)$, and $F(L)$ are (possibly) infinite-order square summable polynomials in the lag operator $L{ }^{11}$ The second step in the solution method is to calculate the Wiener-Kolmogorov optimal prediction formula, which yield the conditional expectations ${ }^{12}$

$$
\begin{gathered}
\mathbb{E}_{t}^{i}\left(p_{t+1}\right)=L^{-1}\left[G(L)-G_{0}\right] u_{t}+L^{-1}\left[D(L)-D_{0}\right] \varepsilon_{1 t}+L^{-1}\left[F(L)-F_{0}\right] \varepsilon_{2 t} \\
\mathbb{E}_{t}\left(c_{t+1}\right)=L^{-1}\left[C(L)-C_{0}\right] u_{t} .
\end{gathered}
$$

Imposing market clearing (2.4) and rearranging, one obtains

$$
\begin{gathered}
\varphi p_{t}=\int_{0}^{1} \mathbb{E}_{t}\left(p_{t+1}+c_{t+1}\right) d i-s_{t} \\
\varphi\left[G(L) u_{t}+D(L) \varepsilon_{1 t}+F(L) \varepsilon_{2 t}\right]=L^{-1}\left[G(L)-G_{0}\right] u_{t}+L^{-1}\left[D(L)-D_{0}\right] \varepsilon_{1 t} \\
+L^{-1}\left[F(L)-F_{0}\right] \varepsilon_{2 t}+L^{-1}\left[C(L)-C_{0}\right] u_{t}-\left[A(L) \varepsilon_{1 t}+B(L) \varepsilon_{2 t}\right] .
\end{gathered}
$$

The third step of the solution process is to solve the corresponding fixed point problem in the frequency domain. As mentioned above, this is simply for computational convenience. Assuming that this expression holds for all realizations of $u_{t}, \varepsilon_{1 t}$ and $\varepsilon_{2 t}$, the coefficients on $u_{s}, \varepsilon_{1 s}$ and $\varepsilon_{2 s}$ must match for every $s$. In lieu of solving this infinite sequential problem, one can solve an

\footnotetext{
${ }^{11}$ It is important to note that by working in the frequency domain one does not have to take a stance on the explicit functional form of beliefs, $G(L), D(L)$ and $F(L)$. This is especially convenient when beliefs contain moving average components because MA representations are difficult to handle in the time domain.

${ }^{12}$ Note that we can write the demeaned coupon process as $c_{t}=C(L) u_{t}$ where $C(L)=(1-\psi L)^{-1}$.
} 
equivalent functional problem by examining the corresponding power series equalities

$$
\begin{gathered}
\varphi G(z)=z^{-1}\left[G(z)-G_{0}\right]+z^{-1}\left[C(z)-C_{0}\right] \\
\varphi D(z)=z^{-1}\left[D(z)-D_{0}\right]-A(z), \\
\varphi F(z)=z^{-1}\left[F(z)-F_{0}\right]-B(z) .
\end{gathered}
$$

Finding the appropriate functions $G(z), D(z)$, and $F(z)$ in the frequency domain yields the following proposition.

Proposition 1. The candidate equilibrium price is unique and given by

$$
p_{t}=\left[\frac{L A(L)-\varphi^{-1} A\left(\varphi^{-1}\right)}{1-\varphi L}\right] \varepsilon_{1 t}+\left[\frac{L B(L)-\varphi^{-1} B\left(\varphi^{-1}\right)}{1-\varphi L}\right] \varepsilon_{2 t}+\left[\frac{C\left(\varphi^{-1}\right)-C(L)}{1-\varphi L}\right] u_{t},
$$

Proof. Due to the symmetry of the problem and the assumption that the shocks are not correlated, we can focus on solving the fixed-point problem for one process, say $\varepsilon_{1 t}$. A little algebra gives

$$
D(z)(1-\varphi z)=D_{0}+z A(z)
$$

As mentioned above, solutions to the model will be sought in the space spanned by square summable linear combinations of the underlying fundamental shocks, and thus it must be the case that the coefficients $D_{j}$ are square summable. The requirement of square-summability in the time domain corresponds to the requirement that $D(z)$ be analytic on the open unit disk $|z|<1$ in the frequency domain. Given $\varphi>1$, this function will not be analytic unless the free parameter $D_{0}$ removes the singularity at $z=\varphi^{-1}$. This is achieved by setting the residue equal to zero and solving for $D_{0}$, which yields

$$
\begin{gathered}
\lim _{z \rightarrow \varphi^{-1}} D(z)(1-\varphi z)=D_{0}+\varphi^{-1} A\left(\varphi^{-1}\right)=0 \\
D_{0}=-\varphi^{-1} A\left(\varphi^{-1}\right) .
\end{gathered}
$$

This implies $D(z)$ is unique and given by

$$
D(z)^{*}=\frac{z A(z)-\varphi^{-1} A\left(\varphi^{-1}\right)}{(1-\varphi z)}
$$

and thus the candidate equilibrium price process has the form (3.4).

The candidate equilibrium price involves three instances of the prediction formula of Hansen and Sargent (1980), and notice that equations (3.5) and (3.4) specify the candidate equilibrium as a function of the fundamental processes of the model. These cross-equation restrictions are the hallmark of rational expectations models.

The fourth and final step in the solution procedure is to determine whether the ex ante informational assumptions support such a price process. To do so, we construct the observer system 
for agent $i$, which includes all of the conditioning variables on the left hand side and fundamental shocks on the right hand side. Setting this system up for the symmetric case one obtains,

$$
\left[\begin{array}{c}
c_{t} \\
s_{t} \\
p_{t}
\end{array}\right]=\left[\begin{array}{ccc}
C(L) & 0 & 0 \\
0 & A(L) & B(L) \\
\frac{C\left(\varphi^{-1}\right)-C(L)}{1-\varphi L} & \frac{L A(L)-\varphi^{-1} A\left(\varphi^{-1}\right)}{1-\varphi L} & \frac{L B(L)-\varphi^{-1} B\left(\varphi^{-1}\right)}{1-\varphi L}
\end{array}\right]\left[\begin{array}{c}
u_{t} \\
\varepsilon_{1 t} \\
\varepsilon_{2 t}
\end{array}\right]
$$

or more compactly

$$
\mathbf{y}_{t}=\mathcal{M}(L) \boldsymbol{\epsilon}_{t}
$$

If this system is invertible in nonnegative powers of $L$, so that $\epsilon_{t}$ may be obtained as a squaresummable linear combination of current and past $\mathbf{y}_{t}$, then (3.6) is a REE. This suggests that if agents are equipped with basic multivariate statistical analysis (e.g., VAR analysis), then knowledge of $\mathbf{y}_{t}$ is equivalent to knowledge of $\boldsymbol{\epsilon}_{t}$.

In order to proceed, we need to place more structure on the model; anticipating analysis below, we follow Singleton in the specification: ${ }^{13}$

Assumption 1. The supply and coupon processes are given by:

$$
C(L)=\frac{1}{(1-\psi L)}, \quad A(L)=\frac{1}{(1-\rho L)}, \quad B(L)=1+\varsigma L, \quad 0 \leq|\psi|,|\rho|,|\varsigma| \leq 1
$$

Note that under this assumption, supply is the sum of a first-order autoregression $(\mathrm{AR}(1))$ and a first-order moving average $(\mathrm{MA}(1))$. Depending on the relative sizes of $\sigma_{\varepsilon_{1}}^{2}$ and $\sigma_{\varepsilon_{2}}^{2}$ and whether $\rho$ and $\varsigma$ are nonzero, this is general enough to include the following special cases for the univariate representation of supply: white noise, $\mathrm{MA}(1), \operatorname{AR}(1)$ (in the limit as $\sigma_{\varepsilon_{2}}^{2} / \sigma_{\varepsilon_{1}}^{2} \rightarrow \infty$ ), $\operatorname{ARMA}(1,1)$ and $\operatorname{ARMA}(2,1)$. It is now possible to determine conditions for the invertibility of (3.6).

Lemma 1. The vector moving average representation (3.6) is invertible (making (3.4) a REE) provided

$$
\left|\frac{\alpha \varsigma \rho}{\alpha(\rho-\varsigma)+\varsigma \rho}\right| \leq 1
$$

Proof. A necessary and sufficient condition for (3.6) to be a fundamental (Wold) representation for $\left[c_{t}, s_{t}, p_{t}\right]^{\prime}$ and thus invertible, is that the determinant of $\mathcal{M}(z)$ be analytic and have no zeros inside the open unit disk. By direct calculation,

$$
\begin{aligned}
\operatorname{det} \mathcal{M}(z) & =C(z)\left[\frac{A(z)\left[z B(z)-\alpha^{-1} B\left(\alpha^{-1}\right)\right]}{(1-\alpha z)}-\frac{B(z)\left[z A(z)-\alpha^{-1} A\left(\alpha^{-1}\right)\right]}{(1-\alpha z)}\right]=0 \\
& =\frac{\alpha^{-2}[\alpha(\rho-\varsigma)+\varsigma \rho+\alpha \varsigma \rho z]}{(\alpha-\rho)(1-\rho z)} ;
\end{aligned}
$$

\footnotetext{
${ }^{13}$ The model was also solved with $A(L)=1+\phi L$. Adopting a different representation for $A(L)$ will alter the solution of the model slightly but the main results found here continue to hold given $|\phi|<1$.
} 
the stated condition guarantees that det $\mathcal{M}(z)$ does not contain any zeros inside the unit circle, and therefore (3.6) is invertible. ${ }^{14}$

Invertibility makes (3.6) a fundamental (Wold) representation. This is important because it implies $\mathcal{M}(L)$ has a one-sided inverse in nonnegative powers of $L$, so a corollary of the above lemma is that $H_{c}(t) \bigvee H_{s}(t) \bigvee H_{p}(t) \equiv H_{u}(t) \bigvee H_{\varepsilon_{1}}(t) \bigvee H_{\varepsilon_{2}}(t)$. Thus, the observables $c_{t}$, $s_{t}$ and $p_{t}$ span the same linear space as the underlying fundamental shocks $u_{t}, \varepsilon_{1 t}$ and $\varepsilon_{2 t}$, and therefore the Hilbert spaces generated by $\left\{\mathbf{y}_{t}, \mathbf{y}_{t-1}, \mathbf{y}_{t-2}, \ldots\right\}$ and $\left\{\boldsymbol{\epsilon}_{t}, \boldsymbol{\epsilon}_{t-1}, \boldsymbol{\epsilon}_{t-2}, \ldots\right\}$ must be identical (in mean-square sense). Thus, the equality

$$
\mathbb{E}_{t}^{i}\left(p_{t+1}\right)=\Pi\left[p_{t+1} \mid H_{c}(t) \bigvee H_{p}(t) \bigvee H_{s}(t)\right]=\Pi\left[p_{t+1} \mid H_{u}(t) \bigvee H_{\varepsilon_{1}}(t) \bigvee H_{\varepsilon_{2}}(t)\right]
$$

holds for all $i$. By allowing traders to guess an equilibrium price that is a linear combination of the underlying shocks (3.3), equality (3.9) was implicitly assumed to hold. This suggests, and was subsequently proven, that by observing the combination of the history of the coupon process, net supplies and equilibrium prices and having knowledge of VAR analysis, agents would be able to infer the underlying shocks. Of course this relationship does not have to hold in equilibrium. The next section studies whether disparate expectations are preserved in this setup under Singleton's (1987) information structure.

\subsection{Asymmetric Information}

Following the framework of the U.S. bond market, in which there is little uncertainty concerning the coupon stream, Singleton introduced asymmetric information via the supply process. ${ }^{15}$ Suppose there are two distinct groups of traders, in proportion $k$ and $(1-k)$. Traders are not able to observe net supply directly, but every trader receives a private, noisy signal on $A(L) \varepsilon_{1 t}$ and a public signal on $B(L) \varepsilon_{2 t}{ }^{16}$ Denote the two signals by

$$
v_{i t}=A(L) \varepsilon_{1 t}+\eta_{i t}, \quad v_{t}=B(L) \varepsilon_{2 t}
$$

where $\eta_{i t}$ is assumed to be i.i.d, normally distributed with finite variance and is uncorrelated with all other shocks. Not only will each individual trader receive different realizations of $\eta$, but the two groups of traders differ in their qualities of information. Traders in group 1 (in proportion $\mathrm{k}$ ) see private signals with smaller variance $\left(\sigma_{\eta 1}^{2}<\sigma_{\eta 2}^{2}\right)$. Of course agents will also be able to condition

\footnotetext{
${ }^{14}$ Notice that if $\varsigma=0$, representation (3.6) will always be invertible.

${ }^{15} \mathrm{It}$ is important to note that the main results of the paper continue to hold if asymmetric information is introduced via the dividend stream, as is typically the case.

${ }^{16}$ Assuming traders observe a public signal does not imply that traders observe contemporaneous realizations of the shock $\varepsilon_{2 t}$. The assumption of a public signal on $\varepsilon_{2}$ is driven by the Singleton-Townsend truncation technique, which assumes all traders observe $\varepsilon_{2}$ with a two-period lag. The results of this section would continue to hold even if $B(L)=L^{n}$, where $n$ is a finite integer.
} 
on current and past prices, and the information set of agent $i$ at time $t$ is given by

$$
\Omega_{t}^{i}=\left\{c_{t-j}, v_{t-j}, v_{i t-j}, p_{t-j}: j \geq 0\right\}
$$

The central question is, will this heterogenous information be enough to generate and preserve disparate expectations in equilibrium? The exogenous information structure is asymmetric; each investor receives a different realization of $\eta_{t}$ and the two groups receive different qualities of information on average. However, in equilibrium, each trader will also extract information from current and past prices. If the equilibrium price provides a rich enough information structure to bridge the gap among traders, then the equilibrium will degenerate into the one studied in Section 3.2

The difference between this section and the previous section is that the agents must first solve the signal extraction problem, which relates the signals to the underlying shocks. After solving this problem, agents will then use this information to generate a guess of the equilibrium price process. Consider the following signal system for agent $i$,

$$
\left[\begin{array}{c}
v_{i t} \\
v_{t}
\end{array}\right]=\left[\begin{array}{ccc}
A(L) & 1 & 0 \\
0 & 0 & B(L)
\end{array}\right]\left[\begin{array}{l}
\varepsilon_{1 t} \\
\eta_{i t} \\
\varepsilon_{2 t}
\end{array}\right]
$$

or more compactly,

$$
\mathbf{v}_{t}=\mathcal{H}(L) \nu_{t}
$$

Since there are more shocks than observables, agent $i$ will not be able to "see" or infer both economically fundamental shocks $\left(\varepsilon_{1 t}, \varepsilon_{2 t}\right)$. This implies, a priori, that a relationship analogous to (3.9) will not hold. That is,

$$
E_{t}^{i}\left(p_{t+1}\right)=\Pi\left[p_{t+1} \mid H_{c}(t) \bigvee H_{v_{i}}(t) \bigvee H_{v}(t)\right] \neq \Pi\left[p_{t+1} \mid H_{u}(t) \bigvee H_{\varepsilon_{1}}(t) \bigvee H_{\varepsilon_{2}}(t)\right]
$$

So what will the agents be able to infer? To give a concrete example, I adopt Assumption 1 and seek a fundamental representation to replace (3.11). The fundamental representation will then be used by the traders to form a guess of the equilibrium price. The following proposition finds the unique fundamental representation to replace (3.11)

Lemma 2. The fundamental signal system for agent $i$ in group $k$ is given by

$$
\left[\begin{array}{l}
v_{i t} \\
v_{t}
\end{array}\right]=\left[\begin{array}{cc}
\mathcal{J}_{1}(L) & 0 \\
0 & B(L)
\end{array}\right]\left[\begin{array}{l}
\xi_{i t} \\
\varepsilon_{2 t}
\end{array}\right]
$$

where $\mathcal{J}_{1}(L)=\frac{1-\lambda_{1} L}{1-\rho L}$

Proof. We seek a fundamental (Wold) representation for the private signal $\left(v_{i t}\right)$ - call it $\mathcal{J}_{1}(L) \xi_{i t}$. This is accomplished by factorization of the covariance generating function of the private signal, 
which is given by ${ }^{17}$

$$
g_{v_{i}}(z)=\frac{\sigma_{\varepsilon_{1}}^{2}}{(1-\rho z)\left(1-\rho z^{-1}\right)}+\sigma_{\eta_{1}}^{2} .
$$

In establishing a fundamental representation, we seek a $\lambda_{1}$ and $\sigma_{\xi_{1}}^{2}$ such that

$$
\sigma_{\varepsilon_{1}}^{2}+\sigma_{\eta_{1}}^{2}(1-\rho z)\left(1-\rho z^{-1}\right)=\sigma_{\xi_{1}}^{2}\left(1-\lambda_{1} z\right)\left(1-\lambda_{1} z^{-1}\right)
$$

Setting $\lambda_{1}$ equal to the smaller root ensures $\left|\lambda_{1}\right|<1$ and yields ${ }^{18}$

$$
\lambda_{1}=\frac{1}{2}\left[\left(\frac{\sigma_{\varepsilon_{1}}^{2}}{\sigma_{\eta_{1}}^{2} \rho}\right)+\left(\frac{1}{\rho}+\rho\right)-\left\{\left[\frac{\sigma_{\varepsilon_{1}}^{2}}{\sigma_{\eta_{1}}^{2} \rho}+\left(\frac{1}{\rho}+\rho\right)\right]^{2}-4\right\}^{1 / 2}\right] .
$$

The variance $\sigma_{\xi_{1}}^{2}$ is then found by the formula

$$
\sigma_{\xi_{1}}^{2}=\frac{g_{z}(1)}{\mathcal{J}_{1}(1)^{2}}=\frac{\sigma_{\varepsilon_{1}}^{2}+\sigma_{\eta_{1}}^{2}(1-\rho)^{2}}{\left(1-\lambda_{1}\right)^{2}}
$$

Note that Type 2 investors will have an analogous fundamental representation $\mathcal{J}_{2}(L) \xi_{\text {it }}$ with $\lambda_{2}$ and $\sigma_{\xi_{2}}^{2}$ each a function of $\sigma_{\eta_{2}}^{2}$.

This factorization puts the signal in a form that the agents can use to predict next period's price and it also tells us the relationship between the signals and the fundamental shocks. By construction, (3.13) a fundamental (Wold) representation and can now be used to generate trader $i$ 's guess of the equilibrium price.

Corollary 1. Trader i's belief of the current price is given by

$$
p_{t}=D_{i}(L) \xi_{i t}+F(L) \varepsilon_{2 t}+G(L) u_{t}
$$

where $\xi_{i t}$ is related to the underlying shock $\varepsilon_{1 t}$ by the equation

$$
\xi_{i t}=\mathcal{J}_{1}(L)^{-1} A(L) \varepsilon_{1 t}+\mathcal{J}_{1}(L)^{-1} \eta_{i t}=\frac{\varepsilon_{1 t}}{1-\lambda_{1} L}+\frac{1-\rho L}{1-\lambda_{1} L} \eta_{i t} .
$$

Note that as the signal-to-noise ratio $\left(\sigma_{\varepsilon_{1}} / \sigma_{\eta_{j}}\right)$ approaches infinity, $\lambda_{j}$ approaches zero and the first component of (3.15) approaches $\varepsilon_{1 t}$. Conversely as the signal-to-noise ratio approaches zero, $\lambda_{j}$ approaches $\rho$. Therefore traders in group 1, who receive a more precise signal, will have a more accurate guess of the price process on average. Further, because the entire price sequence is observable in equilibrium, in order that agents have perceptions about the serial correlation

\footnotetext{
${ }^{17}$ See Whittle (1983) or Sargent (1987) Chapter XI Section 18.

${ }^{18}$ This also ensures $\xi_{i t}$ lies in the linear space spanned by current and lagged $v_{i t}$ 's. In other words, that $\xi_{i t}$ is the one step ahead prediction error of predicting $v_{i t}$ from its own past, $\xi_{i t}=\Pi\left(v_{i t} \mid v_{i t-1}, v_{i t-2}, \ldots\right)$; and therefore the Hilbert spaces generated by $\left\{v_{i t}, v_{i t-1}, \ldots\right\}$ and $\left\{\xi_{i t}, \xi_{i t-1}, \ldots\right\}$ are equivalent.
} 
properties of prices that are consistent with what is observed, the $D_{i}(L)$ functions must be identical within groups and proportional across groups:

$$
\text { for } i \leq k: \quad D_{i}(L)=\chi_{1} D(L), \quad \text { for } k \leq i: \quad D_{i}(L)=\chi_{2} D(L)
$$

Still, this information setup implies disparate expectations for every $i$

$$
\mathbb{E}_{t}^{i}\left(p_{t+1}\right)=\Pi\left[p_{t+1} \mid H_{c}(t) \bigvee H_{v_{i}}(t) \bigvee H_{v}(t)\right]=\Pi\left[p_{t+1} \mid H_{u}(t) \bigvee H_{\xi_{i}}(t) \bigvee H_{\varepsilon_{2}}(t)\right]
$$

In particular, for $i \leq k$,

$$
\mathbb{E}_{t}^{i} p_{t+1}=L^{-1}\left[D(L)-D_{0}\right] \chi_{1} \xi_{i t}+L^{-1}\left[F(L)-F_{0}\right] \varepsilon_{2 t}+L^{-1}\left[G(L)-G_{0}\right] u_{t}
$$

Given that the $\eta_{i t}$ 's are i.i.d., I will assume that a version of the strong law of large numbers holds for each of the two groups so that the overall impact of the idiosyncratic shocks averages to zero. That is,

$$
E_{t}^{1} p_{t+1} \equiv \int_{0}^{k} \mathbb{E}_{t}^{i} p_{t+1} d i=k L^{-1}\left[D(L)-D_{0}\right] \xi_{1 t}+k L^{-1}\left[F(L)-F_{0}\right] \varepsilon_{2 t}+k L^{-1}\left[G(L)-G_{0}\right] u_{t}
$$

where

$$
\xi_{1 t}=\chi_{1} \int_{0}^{k} \xi_{i t} d i=\frac{\chi_{1} \varepsilon_{1 t}}{\left(1-\lambda_{1} L\right)}
$$

and analogously for $k \leq i \leq 1$. This assumption will not by itself lead to a symmetric REE because the traders have different qualities of information $\left(\sigma_{\eta_{1}}^{2}<\sigma_{\eta_{2}}^{2}\right)$ which implies $\xi_{1} \neq \xi_{2}$. It is important to note that $\chi_{1}>\chi_{2}$ and $\lambda_{1}<\lambda_{2}$ implies traders of group 1 have more accurate perceptions and therefore smaller one-step-ahead forecast errors. Thus heterogenous information and disparate expectations now exists across the two groups,

$$
\mathbb{E}_{t}^{1}\left(p_{t+1}\right)=\Pi\left[p_{t+1} \mid H_{\xi_{1}}(t) \bigvee H_{\varepsilon_{2}}(t) \bigvee H_{u}(t)\right] \neq \mathbb{E}_{t}^{2}\left(p_{t+1}\right)=\Pi\left[p_{t+1} \mid H_{\xi_{2}}(t) \bigvee H_{\varepsilon_{2}}(t) \bigvee H_{u}(t)\right]
$$

Because the idiosyncratic information integrates out, the equilibrium price will be a linear function of $\varepsilon_{1 t}$. Thus, $p_{t}=D(L) \varepsilon_{1 t}+F(L) \varepsilon_{2 t}+G(L) u_{t}$, and $\chi_{1}$ and $\chi_{2}$ solve

$$
\sigma_{\varepsilon_{1}}^{2}=\chi_{1}^{2} \sigma_{\xi_{1}}^{2}=\chi_{2}^{2} \sigma_{\xi_{2}}^{2}
$$

i.e.,

$$
\chi_{1}=\left(\frac{\sigma_{\varepsilon_{1}}^{2}\left(1-\lambda_{1}\right)^{2}}{\sigma_{\varepsilon_{1}}^{2}+\sigma_{\eta_{1}}^{2}(1-\rho)^{2}}\right)^{1 / 2} \chi_{2}=\left(\frac{\sigma_{\varepsilon_{1}}^{2}\left(1-\lambda_{2}\right)^{2}}{\sigma_{\varepsilon_{1}}^{2}+\sigma_{\eta_{2}}^{2}(1-\rho)^{2}}\right)^{1 / 2} .
$$


It is straightforward to verify that if $p_{t}$ is of the form $D(L) \varepsilon_{1 t}+F(L) \varepsilon_{2 t}+G(L) u_{t}$, then the average forecast errors $p_{t+1}-\mathbb{E}_{t}^{1} p_{t+1}$ and $p_{t+1}-\mathbb{E}_{t}^{2} p_{t+1}$ are serially correlated. More troubling is the fact that individual forecast errors are also serially correlated. If this serial correlation can be exploited to improve predictions (as will be shown), perceptions (e.g., (3.14)) will not match reality, and we will not have a REE. The extent to which this additional information generated by the candidate price process can be exploited is the crux of the issue. Letting $i$ and $j$ denote the two groups of traders, the market clearing condition is

$$
\begin{gathered}
\varphi p_{t}=\int_{0}^{k} \mathbb{E}_{t}^{i}\left(p_{t+1}+c_{t+1}\right) d i+\int_{k}^{1} \mathbb{E}_{t}^{j}\left(p_{t+1}+c_{t+1}\right) d j-s_{t} \\
\varphi\left[D(L) \varepsilon_{1 t}+F(L) \varepsilon_{2 t}+G(L) u_{t}\right]=k L^{-1}\left[D(L)-D_{0}\right] \frac{\varepsilon_{1 t} \chi_{1}}{1-\lambda_{1} L}+(1-k) L^{-1}\left[D(L)-D_{0}\right] \frac{\varepsilon_{1 t} \chi_{2}}{1-\lambda_{2} L} \\
+L^{-1}\left[F(L)-F_{0}\right] \varepsilon_{2 t}+L^{-1}\left[G(L)-G_{0}\right] u_{t}+L^{-1}\left[C(L)-C_{0}\right] u_{t}-A(L) \varepsilon_{1 t}-B(L) \varepsilon_{2 t} .
\end{gathered}
$$

It is easy to see that the solution for $F(z)$ and $G(z)$ will be the same as the symmetric case; specifically,

$$
\begin{gathered}
F(z)^{*}=\frac{z B(z)-\varphi^{-1} B\left(\varphi^{-1}\right)}{1-\varphi z} \\
G(z)^{*}=\frac{C\left(\varphi^{-1}\right)-C(z)}{1-\varphi z} .
\end{gathered}
$$

The third step of the solution procedure is to equate coefficients on $\varepsilon_{1 t}, \varepsilon_{1 t-1}, \ldots$, which yields the power series equality

$$
\begin{gathered}
D(z)\left[-\lambda_{1} \lambda_{2} \varphi z^{3}+\left(\lambda_{2}+\lambda_{1}\right) \varphi z^{2}-\left((1-k) \lambda_{1} \chi_{2}+\lambda_{2} \chi_{1} k+\varphi\right) z+k \chi_{1}+(1-k) \chi_{2}\right] \\
=D_{0}\left[\left(1-\lambda_{2} z\right) k \chi_{1}+\left(1-\lambda_{1} z\right)(1-k) \chi_{2}\right]+z\left(1-\lambda_{1} z\right)\left(1-\lambda_{2} z\right) A(z) .
\end{gathered}
$$

The roots of the cubic equation

$$
-\lambda_{1} \lambda_{2} \varphi z^{3}+\left(\lambda_{2}+\lambda_{1}\right) \varphi z^{2}-\left(\lambda_{2} \chi_{1} k+(1-k) \lambda_{1} \chi_{2}+\varphi\right) z+k \chi_{1}+(1-k) \chi_{2}
$$

will determine the uniqueness of the candidate equilibrium encountered. The following proposition shows that for the supply process given by Assumption 1 and assuming the sum of the gross interest rate and supply response exceeds unity $(\varphi=\xi+\alpha>1)$, there is a unique candidate price.

Proposition 2. Given $\varphi>1$ and Assumption 1, the price process generated by information (3.10) and the market clearing condition (3.17) is unique.

Proof. See Appendix B

In order for the equilibrium price to be unique, we need exactly one root of (3.18) to lie inside the unit circle. If no root lies inside the unit circle, then the free parameter $D_{0}$ will not be pinned down and $D(z)$ will not be unique. If more than one root lies inside the unit circle, a square-summable 
$D(z)$ satisfying (3.18) does not exist. The proof found in Appendix B shows that there exists exactly one root that lies inside the unit circle. Let $\theta$ denote this root. Then $D_{0}$ will be set to remove this singularity as before; in this case we have

$$
D_{0}^{*}=-\frac{\theta\left(1-\lambda_{1} \theta\right)\left(1-\lambda_{2} \theta\right) A(\theta)}{\left(1-\lambda_{2} \theta\right) k \chi_{1}+\left(1-\lambda_{1} \theta\right)(1-k) \chi_{2}}
$$

Substituting $D_{0}^{*}$ into $D(z)$

$$
\begin{gathered}
D(z)\left[-\lambda_{1} \lambda_{2} \varphi z^{3}+\left(\lambda_{2}+\lambda_{1}\right) \varphi z^{2}-\left((1-k) \lambda_{1} \chi_{2}+\lambda_{2} \chi_{1} k+\varphi\right) z+k \chi_{1}+(1-k) \chi_{2}\right] \\
=\frac{-\left(\theta\left(1-\lambda_{1} \theta\right)\left(1-\lambda_{2} \theta\right) A(\theta)\right)\left[\left(1-\lambda_{2} z\right) k \chi_{1}+\left(1-\lambda_{1} z\right)(1-k) \chi_{2}\right]}{\left(\left(1-\lambda_{2} \theta\right) k \chi_{1}+\left(1-\lambda_{1} \theta\right)(1-k) \chi_{2}\right)}+z\left(1-\lambda_{1} z\right)\left(1-\lambda_{2} z\right) A(z)
\end{gathered}
$$

or more compactly

$$
D(L)^{*} \varepsilon_{1 t}=\left[\frac{D_{0}^{*}\left[\left(1-\lambda_{2} L\right) k \chi_{1}+\left(1-\lambda_{1} L\right)(1-k) \chi_{2}\right]+L\left(1-\lambda_{1} L\right)\left(1-\lambda_{2} L\right) A(L)}{-\lambda_{1} \lambda_{2} \varphi L^{3}+\left(\lambda_{2}+\lambda_{1}\right) \varphi L^{2}-\left((1-k) \lambda_{1} \chi_{2}+\lambda_{2} \chi_{1} k+\varphi\right) L+k \chi_{1}+(1-k) \chi_{2}}\right] \varepsilon_{1 t} .
$$

We now have our unique candidate equilibrium price process,

$$
p_{t}=D(L)^{*} \varepsilon_{1 t}+F(L)^{*} \varepsilon_{2 t}+G(L)^{*} u_{t}
$$

and we can construct the post-equilibrium observer system for a trader $i$ :

$$
\begin{gathered}
{\left[\begin{array}{c}
c_{t} \\
v_{i t} \\
v_{t} \\
p_{t}
\end{array}\right]=} \\
{\left[\begin{array}{cccc}
C(L) & 0 & 0 & 0 \\
0 & A(L) & 0 & 1 \\
0 & 0 & B(L) & 0 \\
G(L)^{*} & D(L)^{*} & F(L)^{*} & 0
\end{array}\right]\left[\begin{array}{c}
u_{t} \\
\varepsilon_{1 t} \\
\varepsilon_{2 t} \\
\eta_{i t}
\end{array}\right]} \\
\mathbf{v}_{t}^{\prime}=\mathcal{H}(L) \boldsymbol{\epsilon}_{t}
\end{gathered}
$$

Representation (3.21) corresponds to the information available to trader $i$ at time $t, \Omega_{t}^{i}$. If $(3.21)$ is a fundamental (Wold) representation, then the Hilbert spaces spanned by $\left\{\mathbf{v}_{t}^{\prime}, \mathbf{v}_{t-1}^{\prime}, \ldots\right\}$ and $\left\{\boldsymbol{\epsilon}_{t}, \boldsymbol{\epsilon}_{t-1}, \ldots\right\}$ are equivalent, and every trader will be able to infer the shock $\varepsilon_{1 t}$ by observing the price sequence. In other words, even though we assumed (at (3.14)) that agents saw only a noisy signal on $\varepsilon_{1 t}$ when forming expectations, the market interactions of those agents injects enough information into the price to reveal the underlying $\varepsilon_{1 t}$ process. Thus after conditioning on the current price, every investor will have identical forecasts in equilibrium. This leads to the following proposition.

Proposition 3. Under Assumption 1, the candidate price process (3.20) reveals the current and past realizations of the fundamental shocks $\left\{\varepsilon_{1 t}\right\},\left\{\varepsilon_{2 t}\right\}$ and $\left\{u_{t}\right\}$ to all traders. 
Proof. The proof is given in Appendix B. The intuition is as follows.

Following Lemma 1, in order to prove that (3.21) is fundamental and reveals $u_{t}, \varepsilon_{1 t}$ and $\varepsilon_{2 t}$, we must show that the determinant of $\mathcal{H}(z)$ has no zeros inside the unit circle. This implies that traders equipped with VAR analysis will be able to figure out the underlying economic shocks of the economy. Recall $B(z)=1+\varsigma z$ where $|\varsigma|<1$. By direct calculation, the numerator of $\operatorname{det} \mathcal{H}(z)$ is

$$
-(1+\varsigma z)(z-\theta)\left[Q z^{2}-R z+S\right]
$$

where

$$
\begin{gathered}
Q=\lambda_{1} \lambda_{2}\left[k \chi_{1}\left(1-\lambda_{2} \theta\right)+\chi_{2}(1-k)\left(1-\lambda_{1} \theta\right)\right](1-\theta \rho) \\
R=\left(1-\lambda_{1} \theta\right)\left(1-\lambda_{2} \theta\right)\left[k \chi_{1} \lambda_{2}+(1-k) \chi_{2} \lambda_{1}\right]+(1-\theta \rho)\left[k \chi_{1} \lambda_{1}\left(1-\lambda_{2} \theta\right)+(1-k) \chi_{2} \lambda_{2}\left(1-\lambda_{1} \theta\right)\right] \\
S=\left(1-\lambda_{1} \theta\right)\left(1-\lambda_{2} \theta\right)\left[k \chi_{1}+(1-k) \chi_{2}\right] .
\end{gathered}
$$

The determinant of $\mathcal{H}(z)$ has four zeros. The root $z=\theta$ is by construction (recall $D_{0}^{*}$ was set to ensure such a numerator zero would cancel the like term in the denominator of $D(z)$ ) and the root $z=-\psi^{-1}$ lies outside the unit circle. Therefore, the roots of

$$
Q z^{2}+R z+S
$$

determine whether or not representation (3.21) is a fundamental (Wold) moving average. Appendix B shows that for $\varphi>1$ there exists no roots that lie inside the unit circle.

The upshot is simply that the assumption of asymmetric information is not sustainable in equilibrium. Traders will surely condition on past prices and update their forecasts accordingly. Showing (3.21) is a fundamental representation is tantamount to the argument that all traders will guess a price process of the form

$$
p_{t}=D(L) \varepsilon_{1 t}+F(L) \varepsilon_{2 t}+G(L) u_{t} .
$$

And therefore the idiosyncratic shock will not enter the average traders' perceptions. More importantly, the conditional expectation (3.12) will be replaced by

$$
E_{t}^{i}\left(p_{t+1}\right)=\Pi\left[p_{t+1} \mid H_{\varepsilon_{1}}(t) \bigvee H_{\varepsilon_{2}}(t) \bigvee H_{u}(t)\right]
$$

All traders will share the same forecast! Hence there is no need to forecast the forecasts of others. This economy will then degenerate into the one studied in Section 3.2. 


\subsection{Townsend-Singleton Truncation Technique}

The results derived here shed light on the consequences of the truncation approach of Townsend (1983) and Singleton (1987), who assumed the equilibrium would preserve disparate expectations and allowed traders to see the fundamental shocks with a two-period lag, that is $\left\{\varepsilon_{1, t-2}, \varepsilon_{1, t-3}, \ldots\right\}$ and $\left\{\varepsilon_{2, t-2}, \varepsilon_{2, t-3}, \ldots\right\} .{ }^{19}$ Therefore instead of matching the entire sequence for $D(z)$ and $F(z)$, the truncation approach requires that one match only a handful of coefficients, namely $D_{0}, D_{1}, F_{0}$ and $F_{1}$. This, however, only approximates the actual equilibrium. To see this more clearly, note that the privately held information of each trader under this informational assumption is given by

$$
\begin{gathered}
{\left[\begin{array}{c}
\varepsilon_{1, t-2} \\
\varepsilon_{2, t-2} \\
v_{i t}
\end{array}\right]=\left[\begin{array}{ccc}
L^{2} & 0 & 0 \\
0 & L^{2} & 0 \\
A(L) & 0 & 1
\end{array}\right]\left[\begin{array}{l}
\varepsilon_{1 t} \\
\varepsilon_{2 t} \\
\eta_{i t}
\end{array}\right]} \\
\mathbf{x}_{t}=\mathcal{N}(L) \boldsymbol{\epsilon}_{t}
\end{gathered}
$$

The above representation is not a fundamental one due to the zero in the determinant of $\mathcal{N}(L)$ at $L=0$; therefore traders will not be able to infer the economically fundamental shocks $\varepsilon_{1 t}$ and $\varepsilon_{2 t}$. This signal extraction problem is slightly different from the one encountered in the previous section and is an artifact of the truncation approach. In order to obtain a fundamental representation, we must employ Blaschke matrices (see Hansen and Sargent (1991)). The following lemma shows how Blaschke matrices are used to derive the fundamental representation.

Lemma 3. The fundamental representation of (3.24) is given by

$$
\mathbf{x}_{t}=\mathcal{N}^{*}(L) \boldsymbol{\epsilon}_{t}^{*}
$$

where

$$
\begin{gathered}
\mathcal{N}^{*}(L)=\hat{\mathcal{N}}(L) \mathbf{W} \mathcal{B}(L), \quad \boldsymbol{\epsilon}_{t}^{*}=\mathcal{B}\left(L^{-1}\right) \mathbf{W}^{\prime} \boldsymbol{\epsilon}_{t} \\
\hat{\mathbf{M}}(\mathbf{L})=\left[\begin{array}{ccc}
L^{2} \sigma_{\varepsilon_{1}} & 0 & 0 \\
0 & L^{2} \sigma_{\varepsilon_{2}} & 0 \\
A(L) \sigma_{\varepsilon_{1}} & 0 & \sigma_{\eta_{j}}
\end{array}\right] \quad \mathbf{W}=\left[\begin{array}{ccc}
-\frac{\sigma_{\eta_{j}}}{\sqrt{A_{0}^{2} \sigma_{\varepsilon_{1}}^{2}+\sigma_{\eta_{j}}^{2}}} & 0 & \frac{\sigma_{\varepsilon_{1}}^{2} A_{0}}{\sqrt{A_{0}^{2} \sigma_{\varepsilon_{1}}^{2}+\sigma_{\eta_{j}}^{2}}} \\
0 & 1 & 0 \\
\frac{\sigma_{\varepsilon_{1}}^{2} A_{0}}{\sqrt{A_{0}^{2} \sigma_{\varepsilon_{1}}^{2}+\sigma_{\eta_{j}}^{2}}} & 0 & \frac{\sigma_{\eta_{j}}}{\sqrt{A_{0}^{2} \sigma_{\varepsilon_{1}}^{2}+\sigma_{\eta_{j}}^{2}}}
\end{array}\right] \quad \mathbf{B}(\mathbf{L})=\left[\begin{array}{ccc}
L^{-2} & 0 & 0 \\
0 & L^{-2} & 0 \\
0 & 0 & 1
\end{array}\right]
\end{gathered}
$$

and $j=1,2$ denoting the two distinct groups of traders.

Proof. See Appendix B.

\footnotetext{
${ }^{19}$ It is assumed that every trader can observe the entire sequence of $\left\{u_{t}\right\}$.
} 
Thus the fundamental innovations $\left(\epsilon_{t}^{*}\right)$ implied by this truncation approach are linear combinations of all the underlying shocks, including the idiosyncratic shock $\eta_{i t}$. It is obvious, due to the law of large numbers, that the market-wide average of the equilibrium price will not include $\eta_{i t}$. However the expectations will continue to be disparate across the two groups due to the presence of the variance of $\eta$ in $\mathbf{W}$. Therefore when solving for the undetermined coefficients, the solution will slightly differ from the symmetric information equilibrium (3.4).

Comparing the informational assumption of (3.24) with that of Section 3.3, Equation (3.11) implies less information in that investors are not able to see $\varepsilon_{1 t}$ with a two-period lag. ${ }^{20}$ In fact, the only information given to traders concerning $\varepsilon_{1 t}$ is that which can be extracted from the signal $v_{i t}$. However, through the information contained in the candidate price process $p_{t}$, it was shown that investors actually had the information set of $\left\{\varepsilon_{1 t}, \varepsilon_{1, t-1}, \ldots\right\}$ and $\left\{\varepsilon_{2 t}, \varepsilon_{2, t-1}, \ldots\right\}$, the equilibrium derived in Section 3.1. Therefore assuming that investors could only condition on $\left\{\varepsilon_{1, t-2}, \ldots\right\}$ and $\left\{\varepsilon_{2, t-2}, \ldots\right\}$ only approximated the actual symmetric equilibrium. Indeed, when traders see $\left\{p_{t}\right\}$ in addition to (3.25), then all underlying uncertainty about the fundamental shocks is revealed.

\section{Conclusion}

It seems doubtful that the bulk of fluctuations in asset markets is due primarily to differences in risk tolerance. A more likely alternative is that trade is generated by agents who are endowed with different sets of information. Unfortunately, tractable models of heterogenous information in a dynamic setting are difficult to construct and even more difficult to sustain in equilibrium. This paper has demonstrated explicitly how endogenous variables reveal the privately held information of other agents in a dynamic asset pricing model. It was shown, via frequency domain techniques, that the invertibility of the post-equilibrium observer system corresponds to the revelation of economically fundamental shocks. Traders equipped with basic statistical analysis can then condition upon this "new" information and update their forecasts accordingly. Solution techniques and informational constructs previously believed to maintain asymmetric information were shown in fact to lead to revealing equilibria. The information content of the price process was too rich to sustain disparate information in equilibrium.

An obvious extension of this research is to develop a dynamic rational expectations model that preserves asymmetric information. To that end, Kasa, Walker, and Whiteman (2006) show how this can be achieved in the frequency domain by assuming a special information structure that gives rise to zeros in the post-equilibrium observer equations. These zeros prohibit equilibrium prices from revealing the economically fundamental shocks. Woodford (2003) achieves this goal in the time domain by following Sims (2001) in assuming that agents have limited capacity. That these efforts seem to require very special circumstances to preserve differential information in equilibrium when disparate expectations seem so widespread, suggests that models of asymmetric information will constitute a fruitful area of research for some time to come.

\footnotetext{
${ }^{20}$ The public signal $v_{t}$ could have been lagged by two periods with no change in the fundamental result. This is because all asymmetric information arises from the private signal.
} 


\section{Appendix A: Equilibrium Multiplicity}

Recall the following demand schedule for the risky asset

$$
z_{i t}=\frac{\mathbb{E}_{t}^{i}\left(p_{t+1}+c_{t+1}\right)-\alpha p_{t}}{\gamma \delta_{i}}
$$

where $\delta_{i}=\operatorname{var}_{t}^{i}\left(p_{t+1}+c_{t+1}\right)$ and $\alpha=(1+r)>1$. This appendix shows how the conditional variance term $\delta$ leads to multiple equilibria and the conditions under which the results of Section 3.3 continue to hold. To shorten the length of this appendix, I will assume that supply is given by: $s_{t}=A(L) \varepsilon_{1 t}$, and that traders see only the private signal and the coupon stream, $\Omega_{i}^{t}=\left\{v_{i t-j}, c_{t-j}: j>0\right\}$. Adding the second component of the supply process does not change the results qualitatively.

Consider the same informational setup of as before with the signal system for agent $i<k$ given by

$$
\left[\begin{array}{l}
v_{i t} \\
c_{t}
\end{array}\right]=\left[\begin{array}{cc}
\mathcal{J}_{1}(L) & 0 \\
0 & C(L)
\end{array}\right]\left[\begin{array}{l}
\xi_{i t} \\
u_{t}
\end{array}\right]
$$

Invoking Assumption 1 implies the derivations follow exactly as in Section 3.3, with the only difference being the addition of the conditional variance term, $\delta$. Given that agents initially only condition on the information extracted from signals and that they are able to match the serial correlation properties of the actual price process $\left(p_{t}=D(L) \varepsilon_{1 t}+G(L) u_{t}\right)$, the conditional variance terms will be identical across groups in equilibrium, i.e.,

$$
\begin{aligned}
\delta & =\operatorname{var}_{t}\left(p_{t+1}\right)+\operatorname{var}_{t}\left(c_{t+1}\right)+2 \operatorname{cov}_{t}\left(p_{t+1}, c_{t+1}\right) \\
& =D_{0}^{2} \sigma_{\varepsilon_{1}}^{2}+\left(G_{0}+C_{0}\right)^{2} \sigma_{u}^{2} .
\end{aligned}
$$

The corresponding market-clearing condition is given by

$$
\begin{gathered}
\varphi p_{t}=\int_{0}^{k} \mathbb{E}_{t}^{i}\left(p_{t+1}+c_{t+1}\right) d i+\int_{k}^{1} \mathbb{E}_{t}^{j}\left(p_{t+1}+c_{t+1}\right) d j-\delta \gamma s_{t} \\
\varphi\left[D(L) \varepsilon_{1 t}+G(L) u_{t}\right]=k L^{-1}\left[D(L)-D_{0}\right] \frac{\varepsilon_{1 t} \chi_{1}}{1-\lambda_{1} L}+(1-k) L^{-1}\left[D(L)-D_{0}\right] \frac{\varepsilon_{1 t} \chi_{2}}{1-\lambda_{2} L} \\
+L^{-1}\left[G(L)-G_{0}\right] u_{t}+L^{-1}\left[C(L)-C_{0}\right] u_{t}-\delta \gamma A(L) \varepsilon_{1 t}
\end{gathered}
$$

where now $\varphi=[\gamma \delta \xi+\alpha]>1$. As before, both $D(z)$ and $G(z)$ must be analytic on the unit disk for all realizations of $\varepsilon_{1 t}$ and $u_{t}$, but the inclusion of the conditional variance has linked the two power series, which can be seen by rearranging and expanding the conditional variance term,

$$
\begin{gathered}
D(z)\left[-\lambda_{1} \lambda_{2} \varphi z^{3}+\left(\lambda_{2}+\lambda_{1}\right) \varphi z^{2}-\left((1-k) \lambda_{1} \chi_{2}+\lambda_{2} \chi_{1} k+\varphi\right) z+k \chi_{1}+(1-k) \chi_{2}\right] \\
=D_{0}\left[\left(1-\lambda_{2} z\right) k \chi_{1}+\left(1-\lambda_{1} z\right)(1-k) \chi_{2}\right]+z\left(1-\lambda_{1} z\right)\left(1-\lambda_{2} z\right) \gamma\left[D_{0}^{2} \sigma_{\varepsilon_{1}}^{2}+\left(G_{0}+C_{0}\right)^{2} \sigma_{u}^{2}\right] A(z) \\
G(z)(1-\varphi z)=G_{0}+C_{0}-C(z)
\end{gathered}
$$

There exists a potential pole at $z=\left|\varphi^{-1}\right|<1$ in (4.3) unless the free parameter $G_{0}$ is set to remove the singularity. That is, $G_{0}=C\left(\varphi^{-1}\right)-C_{0}$ and $G^{*}(z)=\frac{C\left(\varphi^{-1}\right)-C(z)}{1-\varphi z}$. The root condition for uniqueness in (4.2) continues to be identical to the previous section (Equation (3.18)), and we know that there exists exactly one root $(\theta)$ that lies inside the unit circle. Then as before, $D_{0}$ must be set to remove the singularity at 
$z=\theta$. Thus we have

$$
D_{0}\left[\left(1-\lambda_{1} \theta\right)(1-k) \chi_{2}+\left(1-\lambda_{2} \theta\right) k \chi_{1}\right]+\left[D_{0}^{2} \sigma_{\varepsilon_{1}}^{2}+C\left(\varphi^{-1}\right)^{2} \sigma_{u}^{2}\right] \gamma A(\theta) \theta\left(1-\lambda_{1} \theta\right)\left(1-\lambda_{2} \theta\right)=0
$$

Given that there are two roots that satisfy (4.4), there are exactly two equilibria. Moreover, the excess 'noise' associated with the stochastic coupon stream coupled with risk-averse investors severely diminishes the probability of finding equilibria that are real valued. In order for $D_{0}$ (and the corresponding price process) to be real valued, restrictions must be placed on the parameter values. Removing uncertainty in the coupon stream $\left(\sigma_{u}^{2}=0\right)$ yields two equilibria with ${ }^{21}$

$$
D_{0}^{*}=\left\{\begin{array}{l}
-\frac{\left(1-\lambda_{1} \theta\right)(1-k) \chi_{2}+\left(1-\lambda_{2} \theta\right) k \chi_{1}}{\sigma_{\varepsilon_{1}}^{2} \gamma A(\theta) \theta\left(1-\lambda_{1} \theta\right)\left(1-\lambda_{2} \theta\right)} \\
0
\end{array}\right.
$$

The second case implies $p_{t}=G^{*}(L) u_{t}$ for all $t$. However, if $\sigma_{u}^{2} \neq 0$, then, from the quadratic formula, a necessary and sufficient condition for finding a real solution is given by the following restriction

$$
\sigma_{u}^{2} \leq \frac{\left[\left(1-\lambda_{1} \theta\right)(1-k) \chi_{2}+\left(1-\lambda_{2} \theta\right) k \chi_{1}\right]^{2}}{4 \sigma_{\varepsilon_{1}}^{2} \gamma^{2} A(\theta)^{2} \theta^{2}\left(1-\lambda_{1} \theta\right)^{2}\left(1-\lambda_{2} \theta\right)^{2} C\left(\varphi^{-1}\right)^{2}}
$$

Assuming $\sigma_{u}^{2}$ satisfies the above restriction then the number of roots in $D_{0}$ satisfying (4.4) will be the number of equilibria encountered. While the potential existence of multiple equilibria is disconcerting, we are more concerned here with the revelation properties of the price process(es). The candidate equilibrium price process(es) will be given by: $p_{t}=D(L)^{*} \varepsilon_{1 t}+G(L)^{*} u_{t}$, where

$$
\begin{gathered}
D(L)^{*}=\frac{D_{0}^{*}\left[\left(1-\lambda_{2} L\right) k \chi_{1}+\left(1-\lambda_{1} L\right)(1-k) \chi_{2}\right]+L\left(1-\lambda_{1} L\right)\left(1-\lambda_{2} L\right) \gamma\left(D_{0}^{* 2} \sigma_{\varepsilon_{1}}^{2}+C\left(\varphi^{-1}\right)^{2} \sigma_{u}^{2}\right) A(L)}{-\lambda_{1} \lambda_{2} \varphi L^{3}+\left(\lambda_{2}+\lambda_{1}\right) \varphi L^{2}-\left((1-k) \lambda_{1} \chi_{2}+\lambda_{2} \chi_{1} k+\varphi\right) L+k \chi_{1}+(1-k) \chi_{2}} \\
G(L)^{*}=\frac{C(\varphi)^{-1}-C(L)}{1-\varphi L}
\end{gathered}
$$

The post-equilibrium observer system for trader $i$ is then

$$
\begin{gathered}
{\left[\begin{array}{c}
v_{i t} \\
c_{t} \\
p_{t}
\end{array}\right]=} \\
{\left[\begin{array}{ccc}
A(L) & 0 & 1 \\
0 & C(L) & 0 \\
D(L)^{*} & G(L)^{*} & 0
\end{array}\right]\left[\begin{array}{c}
\varepsilon_{1 t} \\
u_{t} \\
\eta_{i t}
\end{array}\right]} \\
\mathbf{x}_{t}=\mathcal{Q}(L) \boldsymbol{\epsilon}_{t}
\end{gathered}
$$

As in the previous section, this observer system will reveal the underlying shocks $\varepsilon_{1 t}$ and $u_{t}$ if (4.8) is a fundamental Wold representation; that is, if the determinant of $\mathcal{Q}(L)$ does not contain any zeros inside the unit circle. Assuming the minimum variance condition (4.6) is met with equality implies that there exists exactly one root that solves (4.4),

$$
D_{0}^{*}=-\frac{\left(1-\lambda_{1} \theta\right)(1-k) \chi_{2}+\left(1-\lambda_{2} \theta\right) k \chi_{1}}{2 \sigma_{\varepsilon_{1}}^{2} \gamma A(\theta) \theta\left(1-\lambda_{1} \theta\right)\left(1-\lambda_{2} \theta\right)} .
$$

\footnotetext{
${ }^{21}$ Singleton(1987) found these equilibria numerically.
} 
The price process is therefore unique and given by

$$
\begin{gathered}
D(z)^{*}\left[-\lambda_{1} \lambda_{2} \varphi z^{3}+\left(\lambda_{2}+\lambda_{1}\right) \varphi z^{2}-\left((1-k) \lambda_{1} \chi_{2}+\lambda_{2} \chi_{1} k+\varphi\right) z+k \chi_{1}+(1-k) \chi_{2}\right] \\
=\frac{\left(1-\lambda_{1} \theta\right)(1-k) \chi_{2}+\left(1-\lambda_{2} \theta\right) k \chi_{1}}{2 \gamma \theta^{2} A(\theta)\left(1-\lambda_{1} \theta\right)^{2}\left(1-\lambda_{2} \theta\right)^{2}}\left[z\left(1-\lambda_{1} z\right)\left(1-\lambda_{2} z\right) A(z)\left[\left(1-\lambda_{1} \theta\right)(1-k) \chi_{2}+\left(1-\lambda_{2} \theta\right) k \chi_{1}\right]-\right. \\
\left.\theta\left(1-\lambda_{1} \theta\right)\left(1-\lambda_{2} \theta\right) A(\theta)\left[\left(1-\lambda_{1} z\right)(1-k) \chi_{2}+\left(1-\lambda_{2} z\right) k \chi_{1}\right]\right]
\end{gathered}
$$

Comparing (4.9) with (3.19) it is easy to see how risk aversion enters the candidate equilibrium price process. More importantly, it is easy to see that the zeros of (3.19) and (4.9) will coincide. In other words, the zeros of the determinant of $\mathcal{Q}(L)$ are given by

$$
\begin{aligned}
\operatorname{det} \mathcal{Q}(L) & =-D(L)^{*} C(L) \\
& =-(1+\psi z)(z-\theta)\left[Q z^{2}+R z+S\right]
\end{aligned}
$$

The exact condition for price revelation encountered in Section 3.3! Appendix B shows that there are no zeros that lie inside the unit circle and therefore the price process will reveal all privately held information. The equilibrium price of this economy is determined by a symmetric information structure. Moreover as $\sigma_{u}^{2}$ falls below the minimum variance condition (4.6), the economy converges to the candidate equilibria with $D_{0}^{*}$ given by (4.5). Using the techniques of this paper, it is easy to show that these candidate equilibria will also reveal and degenerate into symmetric information equilibria.

\section{Appendix B: Proof of Propositions}

\section{Proof of Proposition 2}

The following theorem will be used to show that two roots of (3.18) lie outside the unit circle with one real root inside the unit circle (see, Churchill and Brown (1990)). ${ }^{22}$

Theorem A (Rouché). Suppose that two functions $f$ and $g$ are analytic inside and on a simple closed contour $C$. If $|f(z)|>|g(z)|$ at each point on $C$, then the functions $f(z)$ and $f(z)+g(z)$ have the same numbers of zeros, counting multiplicities, inside $C$.

To determine the number of roots of the cubic equation

$$
-\lambda_{1} \lambda_{2} \varphi z^{3}+\left(\lambda_{2}+\lambda_{1}\right) \varphi z^{2}-\left(\lambda_{2} \chi_{1} k+(1-k) \lambda_{1} \chi_{2}+\varphi\right) z+k \chi_{1}+(1-k) \chi_{2}
$$

interior to the circle $|z|=1$, rewrite (4.10) as

$$
-a_{3} z^{3}+a_{2} z^{2}-a_{1} z+a_{0}
$$

and define

$$
f=-a_{1} z+a_{0}, \quad g=-a_{3} z^{3}+a_{2} z^{2}
$$

\footnotetext{
${ }^{22}$ Thanks to Ken Kasa for suggesting Rouché's Theorem in proving Proposition 3.1.
} 
Notice that $\mathrm{f}$ has a (single) root inside the unit circle, since $a_{1}>a_{0}$. To show this define $\vartheta_{i}=\sigma_{\varepsilon_{1}} / \sqrt{\sigma_{\varepsilon_{1}}^{2}+\sigma_{\eta_{i}}^{2}(1-\rho)^{2}}$ and $\chi_{i}=\vartheta_{i}\left(1-\lambda_{i}\right)$ for $i=1,2$, and note

$$
k \chi_{1}\left(1-\lambda_{2}\right)+(1-k) \chi_{2}\left(1-\lambda_{1}\right)=\left(1-\lambda_{1}\right)\left(1-\lambda_{2}\right)\left[k \vartheta_{1}+(1-k) \vartheta_{2}\right]<1<\varphi
$$

Next, notice that $|f|>|g|$ on $|z|=1$; to wit, note that ${ }^{23}$

$$
\left|-\varphi\left(1-\lambda_{1}\right)\left(1-\lambda_{2}\right)\right|<\varphi<\left|\left(1-\lambda_{1}\right)\left(1-\lambda_{2}\right)\left[k \vartheta_{1}+(1-k) \vartheta_{2}\right]-2 \varphi\right|
$$

implies

$$
\left|-\varphi\left(1-\lambda_{1}\right)\left(1-\lambda_{2}\right)\right|<\left|\left(1-\lambda_{1}\right)\left(1-\lambda_{2}\right)\left[k \vartheta_{1}+(1-k) \vartheta_{2}\right]-2 \varphi\right|
$$

which gives the result

$$
\left|-\varphi\left(\lambda_{1} \lambda_{2}-\lambda_{2}-\lambda_{1}\right)\right|<\left|\left(1-\lambda_{1}\right)\left(1-\lambda_{2}\right)\left[k \vartheta_{1}+(1-k) \vartheta_{2}\right]-\varphi\right|
$$

QED

\section{Proof of Proposition 3}

This section shows that the quadratic given by (3.22) has both roots outside of the unit circle. This implies that the representation (3.21) is fundamental, hence eliminating the need to forecast the forecasts of others. Recall the quadratic is given by

$$
\begin{gathered}
Y(z)=Q z^{2}-R z+S \\
Q=\lambda_{1} \lambda_{2}\left[k \chi_{1}\left(1-\lambda_{2} \theta\right)+(1-k) \chi_{2}\left(1-\lambda_{1} \theta\right)\right](1-\theta \rho) \\
R=\left(1-\lambda_{1} \theta\right)\left(1-\lambda_{2} \theta\right)\left[k \chi_{1} \lambda_{2}+(1-k) \chi_{2} \lambda_{1}\right]+(1-\theta \rho)\left[k \chi_{1} \lambda_{1}\left(1-\lambda_{2} \theta\right)+(1-k) \chi_{2} \lambda_{2}\left(1-\lambda_{1} \theta\right)\right] \\
S=\left(1-\lambda_{1} \theta\right)\left(1-\lambda_{2} \theta\right)\left[k \chi_{1}+(1-k) \chi_{2}\right]
\end{gathered}
$$

First notice that $Q, R$ and $S$ are all strictly positive, implying that $Y(0)>0$ and all the corresponding roots must be strictly positive. Moreover $Y(1)>0$; ignoring $(1-k) \chi_{2}$ terms (due to symmetry) yields

$$
\begin{aligned}
Y(1) & =k \chi_{1}\left[\lambda_{1} \lambda_{2}\left(1-\lambda_{2} \theta\right)(1-\theta \rho)-\left(1-\lambda_{1} \theta\right)\left(1-\lambda_{2} \theta\right) \lambda_{2}-(1-\theta \rho) \lambda_{1}\left(1-\lambda_{2} \theta\right)+\left(1-\lambda_{1} \theta\right)\left(1-\lambda_{2} \theta\right)\right] \\
& =k \chi_{1}\left(1-\lambda_{2} \theta\right)\left(1-\lambda_{2}\right)\left[\left(1-\lambda_{1} \theta\right)-\lambda_{1}(1-\theta \rho)\right]
\end{aligned}
$$

which is clearly positive because $\left(1-\lambda_{1} \theta\right)>\lambda_{1}(1-\theta \rho)$.

We now need to rule out the case that both roots lie inside the unit circle. We do this by showing that the minimum lies outside the unit circle.

$$
z^{*}=\operatorname{argmin} Y(z)=\frac{R}{2 Q}>1
$$

\footnotetext{
${ }^{23}$ It is interesting to note in passing that given $\lambda_{1}=\lambda_{2}$ the above inequalities can be established via the unique properties associated with the golden ratio (i.e., the positive number that solves the equation $\phi+\phi^{-1}=1$ ).
} 
Again focusing on $k \chi_{1}$, we need to show that

$$
\begin{aligned}
R-2 Q & =k \chi_{1} \lambda_{2}\left(1-\lambda_{1} \theta\right)\left(1-\lambda_{2} \theta\right)+(1-\theta \rho) k \chi_{1} \lambda_{1}\left(1-\lambda_{2} \theta\right)-2\left[\lambda_{1} \lambda_{2} k \chi_{1}\left(1-\lambda_{2} \theta\right)(1-\theta \rho)\right]>0 \\
& =k \chi_{1}\left(1-\lambda_{2} \theta\right)\left\{\lambda_{2}\left[\left(1-\lambda_{1} \theta\right)-\lambda_{1}(1-\rho \theta)\right]+(1-\rho \theta) \lambda_{1}\left(1-\lambda_{2}\right)\right\}
\end{aligned}
$$

which is clearly positive. QED

\section{Proof of Lemma 3}

Recall from (3.24) that the privately held information is given by the following system

$$
\begin{gathered}
{\left[\begin{array}{c}
\varepsilon_{1, t-2} \\
\varepsilon_{2, t-2} \\
v_{i t}
\end{array}\right]=\left[\begin{array}{ccc}
L^{2} & 0 & 0 \\
0 & L^{2} & 0 \\
A(L) & 0 & 1
\end{array}\right]\left[\begin{array}{l}
\varepsilon_{1 t} \\
\varepsilon_{2 t} \\
\eta_{i t}
\end{array}\right]} \\
\mathbf{x}_{t}=\mathcal{N}(L) \boldsymbol{\epsilon}_{t}
\end{gathered}
$$

The covariance generating function of the above representation is given by

$$
G_{\mathbf{x}}(z)=\mathcal{N}(z) \boldsymbol{\Sigma} \mathcal{N}\left(z^{-1}\right), \quad \boldsymbol{\Sigma}=\left[\begin{array}{ccc}
\sigma_{\varepsilon_{1}}^{2} & 0 & 0 \\
0 & \sigma_{\varepsilon_{2}}^{2} & 0 \\
0 & 0 & \sigma_{\eta_{j}}^{2}
\end{array}\right]
$$

where $j=1,2$ contingent upon the group of investor. Following Rozanov (1967), we can find an equivalent (same covariance generating function) representation through the use of orthogonal matrices $\mathcal{B}(L)$ and $\mathbf{W}$. The corresponding fundamental representation is given by

$$
\mathbf{x}_{t}=\mathcal{N}^{*}(L) \epsilon_{t}^{*}
$$

where

$$
\begin{gathered}
\mathcal{N}^{*}(L)=\hat{\mathcal{N}}(L) \mathbf{W} \mathcal{B}(L), \quad \boldsymbol{\epsilon}_{t}^{*}=\mathcal{B}\left(L^{-1}\right) \mathbf{W}^{\prime} \boldsymbol{\epsilon}_{t} \\
\hat{\mathcal{N}}(L)=\left[\begin{array}{ccc}
L^{2} \sigma_{\varepsilon_{1}} & 0 & 0 \\
0 & L^{2} \sigma_{\varepsilon_{2}} & 0 \\
A(L) \sigma_{\varepsilon_{1}} & 0 & \sigma_{\eta_{j}}
\end{array}\right] \quad \mathbf{W}=\left[\begin{array}{ccc}
-\frac{\sigma_{\eta_{j}}}{\sqrt{A_{0}^{2} \sigma_{\varepsilon_{1}}^{2}+\sigma_{\eta_{j}}^{2}}} & 0 & \frac{\sigma_{\varepsilon_{1}}^{2} A_{0}}{\sqrt{A_{0}^{2} \sigma_{\varepsilon_{1}}^{2}+\sigma_{\eta_{j}}^{2}}} \\
0 & 1 & 0 \\
\frac{\sigma_{\varepsilon_{1}}^{2} A_{0}}{\sqrt{A_{0}^{2} \sigma_{\varepsilon_{1}}^{2}+\sigma_{\eta_{j}}^{2}}} & 0 & \frac{\sigma_{\eta_{j}}}{\sqrt{A_{0}^{2} \sigma_{\varepsilon_{1}}^{2}+\sigma_{\eta_{j}}^{2}}}
\end{array}\right] \quad \mathcal{B}(L)=\left[\begin{array}{ccc}
L^{-2} & 0 & 0 \\
0 & L^{-2} & 0 \\
0 & 0 & 1
\end{array}\right]
\end{gathered}
$$

The matrix $\mathcal{B}(L)$ is a Blaschke matrix that removes the root at zero by "flipping" the root outside the unit circle, while the matrix $\mathbf{W}$ serves to ensure that the Blaschke matrix does not introduce any unwanted poles into the fundamental representation. ${ }^{24}$ It is important to note that the orthogonal properties of the matrices $\mathbf{W}$ and $\mathcal{B}(L)$ (i.e., $\mathbf{W} \mathbf{W}^{\prime}=I$ and $\mathcal{B}(z) \mathcal{B}\left(z^{-1}\right)=I$ ) ensure that the fundamental representation continues to emit the same covariance generating function. This representation is unique up to an orthogonal constant and implies that investors will have knowledge of a linear combination of the innovations $\left(\epsilon_{t}^{*}\right)$.

\footnotetext{
${ }^{24}$ See Lippi and Reichlin (1994) for a good introduction to Blaschke matrices and fundamental representations.
} 


\section{References}

Allen, F., S. Morris, And H. S. Shin (2005): "Beauty Contests and Bubbles," Cowles Foundation Discussion Paper No. 1406. 1, 3, 5

Angeletos, G.-M., And A. Pavan (2005): "Efficient Use of Information and Welfare Analysis in Economies with Complementarities and Asymmetric Information," MIT Working Paper. 1

Bacchetta, P., And E. VAn Wincoop (2004): "Can Information Heterogeneity Explain the Exchange Rate Puzzle?," Working Paper, Studienzentrum Gerzensee. 1, 2

Blanchard, O. J., And C. M. Kahn (1980): "The Solution of Linear Difference Models under Rational Expectations," Econometrica, 48(5), 1305-1312. 5

Brockwell, P. J., And R. A. Davis (1991): Time Series: Theory and Methods. Springer Verlag, New York, second edn. 6

Churchill, R. V., And J. W. Brown (1990): Complex Variables and Applications. McGraw-Hill, New York, fifth edn. 22

Foster, F. D., And S. Viswanathan (1996): "Strategic Trading when Agents Forecast the Forecasts of Others," Journal of Finance, 51(4), 1437-1478. 1

Futia, C. A. (1981): "Rational Expectations in Stationary Linear Models," Econometrica, 49(1), 171-192. 6

Hansen, L. P., And T. J. Sargent (1980): "Formulating and Estimating Dynamic Linear Rational Expectations Models," Journal of Economic Dynamics and Control, 2, 7-46. 9

- (1991): "Two Difficulties in Interpreting Vector Autoregressions," in Rational Expectations Econometrics, ed. by L. P. Hansen, and T. J. Sargent. Westview Press.

He, H., And J. WANG (1995): "Differential Information and Dynamic Behavior of Stock Trading Volume," Review of Financial Studies, 8, 919-972. 1

Hellwig, C., And L. Veldkamp (2005): "Knowing What Others Know: Coordination Motives in Information Acquisition," mimeo, UCLA. 1

KASA, K. (2000): "Forecasting the Forecasts of Others in the Frequency Domain," Review of Economic Dynamics, 3, 726-756. 1, 2, 7

Kasa, K., T. B. Walker, and C. H. Whiteman (2006): "Asset Prices in a Time Series Model with Perpetually Disparately Informed, Competitive Traders," mimeo, University of Iowa. 19

Keynes, J. M. (1936): The General Theory of Employment, Interest and Money. Macmillan, London. 1

LipPi, M., AND L. ReIChlin (1994): "VAR Analysis, Nonfundamental Representations, Blaschke Matrices," Journal of Econometrics, 63, 307-325. 24

LucAs, JR., R. E. (1975): "An Equilibrium Model of the Business Cycle," Journal of Political Economy, 83, 1113-1144. 1 
Morris, S., And H. S. Shin (2003): "Global Games: Theory and Applications," in Advances in Economics and Econometrics: Theory and Applications, ed. by S. T. M. Dewatripont, L.P. Hansen. Cambridge University Press, Cambridge. 1, 3

Muth, J. F. (1960): "Optimal Properties of Exponentially Weighted Forecasts," Journal of American Statistical Association, 55, 299-306. 5

Nimark, K. P. (2005): "Dynamic Pricing, Imperfect Common Knowledge and Inflation Inertia," ECB Working Paper No. 474. 1

Pearlman, J. G., D. Currie, and P. Levine (1986): "Rational Expectations Models with Private Information," Economic Modelling, 3(2), 90-105. 2

Pearlman, J. G., and T. J. Sargent (2005): "Knowing the Forecasts of Others," Review of Economic Dynamics, 8(2), 480-497.

Phelps, Edmund, S. (1983): "The Trouble with 'Rational Expectations' and the Problem of Inflation Stabilization," in Individual Forecasting and Aggregate Outcomes, ed. by R. Frydman, and E. Phelps, pp. 31-45. Cambridge University Press, Cambridge. 1

Rozanov, Y. A. (1967): Stationary Random Processes. Holden-Day, San Francisco. 24

Sargent, T. J. (1987): Macroeconomic Theory. Academic Press, 2 edn. 4

- (1991): "Equilibrium with Signal Extraction from Endogenous Variables," Journal of Economic Dynamics and Control, 15, 245-273. 2, 7

Sims, C. A. (2001): "Implications of Rational Inattention," Working Paper, Princeton University. 19

Singleton, Kenneth, J. (1987): "Asset Prices in a Time Series Model with Disparately Informed, Competitive Traders," in New Approaches to Monetary Economics, ed. by W. Barnett, and K. Singleton. Cambridge University Press, Cambridge. 1, 3

Townsend, R. M. (1983): "Forecasting the Forecasts of Others," Journal of Political Economy, 91, 546588.

Ui, T. (2003): "A Note on the Lucas Model: Iterated Expectations and the Neutrality of Money," Working Paper, Yokohama University. 1

Wang, J. (1993): "A Model of Asset Prices under Asymmetric Information," Review of Economic Studies, $60(2), 249-282.1$

127-168. 1

Whiteman, C. (1983): Linear Rational Expectations Models: A User's Guide. University of Minnesota Press, Minneapolis. 1, 7

Whittle, P. (1983): Prediction and Regulation by Linear Least-Square Methods. University of Minnesota Press, Minneapolis. 13 
Woodford, M. (2003): "Imperfect Common Knowledge and the Effects of Monetary Policy," in Knowledge, Information, and Expectations in Modern Macroeconomics, ed. by P. Aghion, R. Frydman, J. Stiglitz, and M. Woodford. Princeton University Press, Princeton, N.J. 1, 19 